\title{
TREINAMENTO NEUROMOTOR NO PADRÃO DE MARCHA E NA MOBILIDADE DE TORNOZELOS EM IDOSOS
}

\author{
Neuromotor training on gait pattern and mobility of ankles in \\ older people
}
Entrenamiento neuromotor del patrón de marcha y movilidad de tobillos de mayores

Artigo Original

\section{RESUMO}

Objetivo: Analisar o efeito do treinamento neuromotor no padrão de marcha e a mobilidade de tornozelos em idosos. Métodos: Ensaio controlado não aleatorizado, de corte transversal, realizado em Rio Negrinho, Santa Catarina, no período de maio a setembro de 2015, com amostra de 26 idosas divididas em grupo controle $(\mathrm{GC}=15)$ e grupo treinamento neuromotor $(\mathrm{GTN}=11)$. A avaliação do padrão de marcha ocorreu através do Protocolo de Cerny e a mobilidade de tornozelos, através da goniometria. O GC realizou atividade física regular composta por aquecimento, exercícios de alongamento e fortalecimento muscular de grandes grupos musculares de membros e desaquecimento. O GTN recebeu treinamento neuromotor em forma de circuito composto por 10 estações, com aquecimento, treinamento neuromotor, desaquecimento e repetição do circuito em 3 vezes, com permanência de 1 minuto em cada estação e 30 segundos de intervalo entre elas, com progressão de dificuldade dos exercícios após a sexta semana. Ambos os grupos realizaram a atividade por 12 semanas (2 vezes semanais, com duração de 45 minutos). Análise ocorreu pelo teste t, adotando um nível de significância de $p<0,05$. Resultados: Houve melhora significativa em dorsiflexão de ambos os tornozelos (direito $\mathrm{p}=0,00$ e esquerdo $=0,02$ ) e em ambos os grupos; já no padrão de marcha, não houve melhora significativa após treinamento neuromotor (velocidade $p=0,55$; tempo de deambulação $p=0,6$ ). Conclusão: $O$ treinamento neuromotor beneficiou a manutenção do padrão de marcha (velocidade e tempo de deambulação) e a mobilidade articular de tornozelos em idosas avaliadas.

Registro Brasileiro de Ensaios Clínicos: RBR-9tphus

Descritores: Idoso; Marcha; Movimento.

1) Universidade do Contestado - UnC Mafra (SC) - Brasil

\section{ABSTRACT}

Objective: To analyze the effect of neuromotor training on gait pattern and mobility of ankles in older people. Methods: A cross-sectional non-randomized controlled study conducted in Rio Negrinho, Santa Catarina, from May to September 2015 with a sample of 26 older women divided into control group $(C G=15)$ and neuromotor training group (NETG=11). The gait pattern was assessed through the Cerny Protocol and the mobility of ankles through goniometry. The CG performed regular physical activity including warm-up, stretching and strengthening of major muscle groups and cool-down. The NETG performed a neuromotor training as a 10-station circuit including warm-up, neuromotor training, cool-down and repetition of the circuit three times, remaining one minute at each station and keeping a 30-second interval between the stations. The exercise difficulty level increased after the sixth week. Both groups performed the activity for 12 weeks (twice a week for 45 minutes). The analysis was performed using the t-test with a significance level of $p<0.05$. Results: $A$ significant improvement was observed in dorsiflexion of both ankles (right ankle $p=0.00$ and left ankle=0.02) and in both groups; however, the gait pattern did not present a significant improvement after the neuromotor exercise training (speed $p=0.55$; time to ambulation $p=0.6$ ). Conclusion: The neuromotor training maintained the gait pattern (speed and time to ambulation) and the mobility of ankles of the older women assessed.

Descriptors: Aged; Gait; Movement.

Recebido em: 10/12/2015

Revisado em: 19/12/2015

Aceito em: 29/12/2015 


\section{RESUMEN}

Objetivo: Analizar el efecto del entrenamiento neuromotor del patrón de marcha y movilidad de tobillos de mayores. Métodos: Ensayo controlado no aleatorizado, de corte transversal realizado en Rio Negrinho, Santa Catarina, entre mayo y septiembre de 2015 con una muestra de 26 mayores divididas en grupo controle $(G C=15)$ y grupo de entrenamiento neuromotor $(G E N=11)$. La evaluación del patrón de la marcha se dio a través del Protocolo de Cerny y la movilidad de los tobillos a través de la goniometría. En el GC se realizó actividad física regular con calentamiento, ejercicios de estiramiento y de fuerza muscular para los grandes grupos musculares de los miembros y desaceleración. El GEN recibió entrenamiento neuromotor en forma de circuito formado por 10 estaciones con calentamiento, entrenamiento neuromotor, desaceleración y repetición del circuito 3 veces con permanencia de 1 minuto en cada estación y 30 segundos de intervalo entre ellas con progresión de dificultad para los ejercicios después de la sexta semana. Ambos los grupos realizaron la actividad durante 12 semanas (2 veces a la semana, con duración de 45 minutos). El análisis se dio a través del teste T, adoptando un nivel de significancia de $p<0,05$. Resultados: Hubo mejora significativa para la dorsiflexión de ambos los tobillos (derecho $p=0,00$ y izquierdo=0,02) y en ambos los grupos; no hubo mejora significativa después del entrenamiento neuromotor (velocidad $p=0,55$; tiempo de deambulación $p=0,6$ ) en el patrón de la marcha. Conclusión: El entrenamiento neuromotor benefició la manutención del patrón de la marcha (velocidad y tiempo de deambulación) y la movilidad articular de los tobillos de las mayores evaluadas.

Descriptores: Anciano; Marcha; Movimiento.

\section{INTRODUÇÃO}

O envelhecimento populacional é um fenômeno mundial que vem se manifestando de forma rápida e distinta nos países, trazendo grandes desafios para as políticas públicas em assegurar a continuidade do processo de desenvolvimento econômico e social, garantindo a equidade entre os grupos etários na partilha dos recursos, direitos e responsabilidades sociais, bem como um envelhecimento saudável e ativo no controle das doenças crônicas não transmissíveis ${ }^{(1)}$.

A população brasileira, segundo o último censo demográfico (2010), era de 190.755.199 milhões de pessoas. O contingente de pessoas idosas estava em 20.590.599 milhões, aproximadamente $10,8 \%$ da população total. Segundo as perspectivas epidemiológicas, o Brasil, até 2025, passará para a sexta posição mundial em termos de números absolutos ${ }^{(2,3)}$.

A diminuição gradual da capacidade funcional está diretamente relacionada ao processo de envelhecimento e tem uma relação direta e linear com o avançar da idade. Várias capacidades físicas são atingidas, tais como: diminuição da força muscular, flexibilidade, agilidade, coordenação, mobilidade articular e equilíbrio ${ }^{(4-6)}$. A dependência para o desempenho das atividades de vida diária (AVD) tende a aumentar cerca de 5\% na faixa etária de 60 anos para cerca de $50 \%$ entre os com 90 ou mais anos ${ }^{(7)}$.

A eficiência da marcha pode ficar prejudicada com a idade devido a mudanças, como encurtamento e diminuição da altura do passo, alargamento da base de suporte, diminuição da velocidade da marcha e da extensão do joelho e quadril, além do aumento da fase de apoio e do tempo de duplo suporte. Em decorrência disso, os idosos desenvolvem uma marcha com maior gasto energético, o que pode desencadear um declínio das atividades desempenhadas e consequentemente, uma diminuição da força muscular, contribuindo para a modificação da função motora $^{(8)}$.

As alterações no equilíbrio dinâmico e agilidade decorrentes do processo de senescência têm sido minimizadas através de exercícios direcionados para essas valências físicas. Um programa neuromotor aplicado em 100 idosos com idade entre 75 e 80 anos, submetidos a 40 sessões com duração de 50 minutos, com periodicidade de três vezes por semana, demonstrou melhora no equilíbrio e agilidade $^{(9)}$.

Exercícios funcionais também podem ser indicados e apresentar evidências de melhora da força e potência muscular visando a melhora do equilíbrio e independência do idoso ${ }^{(10)}$. Exercícios funcionais de baixa intensidade são capazes de melhorar a capacidade física dos idosos, tornando-os mais independentes ${ }^{(11)}$.

A atividade física regular contendo aquecimento com exercícios de flexibilidade, exercícios de fortalecimento muscular de membros inferiores e tronco, exercícios de equilíbrio, diversos jogos com bolas e desaquecimento com alongamentos demonstrou melhora no equilíbrio estático, mobilidade funcional, bem como subdomínios de funcionamento físico, vitalidade e saúde geral da qualidade de vida em idosos ${ }^{(12)}$.

O treinamento dos mecanismos controladores do equilíbrio estático e dinâmico aumenta a autoconfiança dos idosos, melhora sua capacidade funcional e, consequentemente, reflete na mobilidade articular e no desempenho da marcha ${ }^{(13)}$.

As quedas são um problema de saúde pública entre os idosos, considerando-se a mortalidade, a morbidade e os custos sociais e econômicos delas decorrentes. Além disso, sabe-se que as quedas são importantes fatores causais de aumento no nível de dependência do idoso, pois afetam sua capacidade funcional ${ }^{(14)}$. 
A Organização Mundial da Saúde define o envelhecimento saudável como o processo de desenvolvimento e manutenção da capacidade funcional que permite o bem-estar durante o avançar da idade. A promoção da saúde à população idosa inclui ações de conscientização, implantação e execução da atividade física regular através de estratégias de fortalecimento muscular, alongamento muscular, condicionamento aeróbio e otimização do equilíbrio ${ }^{(15)}$.

A escolha desse tema se deu pela importância da aplicação de exercícios físicos sistematizados em idosos como uma das ferramentas para o desenvolvimento do envelhecimento ativo. $\mathrm{O}$ treinamento neuromotor está entre os exercícios recomendados, pois incorpora habilidades que estimulam os receptores proprioceptivos presentes no corpo, proporcionando a melhora no desempenho físico, equilíbrio, marcha e mobilidade articular de membros inferiores. O estudo poderá contribuir na ampliação dos conteúdos teóricos e práticos da aplicação do treinamento neuromotor em programas voltados aos idosos, quer seja no setor privado ou público.

O objetivo deste estudo foi analisar o efeito do treinamento neuromotor no padrão de marcha e a mobilidade de tornozelos em idosos.

\section{MÉTODOS}

Trata-se de uma pesquisa do tipo ensaio controlado, não aleatorizado, de corte transversal, realizada em Rio Negrinho, Santa Catarina, no período de maio a setembro de 2015.

A amostra contou com idosos do sexo feminino, de 65 a 75 anos, inscritas no Programa de Hipertensão Arterial e Diabetes (HiperDia) da Secretaria Municipal de Saúde de Rio Negrinho/SC, sedentárias e praticantes de atividade física regular com uma frequência de duas vezes semanais. Houve fixação de cartaz nos murais das unidades básica de saúde do município e também o convite oral durante a reunião mensal do Programa HiperDia. Os participantes da reunião mensal do HiperDia perfazem um total de 60 idosos, sendo 6 homens. Das 54 idosas, 15 praticam atividade física regular e 39 idosas eram sedentárias. Após a explicação da pesquisa, 11 idosas sedentárias aceitaram participar do estudo. Os critérios de exclusão envolveram idosos que apresentassem incapacidade física, que utilizassem dispositivo auxiliar para marcha; doenças neurológicas com sequelas; deficiência visual grave; ou qualquer outro distúrbio que pudesse interferir na execução dos exercícios do treinamento neuromotor.

O grupo treinamento neuromotor (GTN) contou com 11 idosas sedentárias e o Grupo Controle (GC) formado por
15 idosas praticantes de atividade física regular com uma frequência de 2 vezes semanais no Grêmio Esportivo Vila Nova da mesma cidade.

A avaliação inicial contou com anamnese, avaliação do padrão de marcha e mobilidade de tornozelos. As informações coletadas na anamnese contaram com perguntas para obter dados sociodemográficos, grau de escolaridade, medicamentos, e histórico de quedas no último ano. Após 12 semanas de aplicação do treinamento neuromotor, ocorreu a reavaliação do padrão de marcha e mobilidade de tornozelos.

Duas pesquisadoras realizaram a anamnese e avaliação do padrão da marcha segundo os protocolos estabelecidos e somente uma pesquisadora realizou a goniometria de tornozelos, pois este teste é examinador-dependente. As avaliações aconteciam a partir de datas pré-agendadas pelas pesquisadoras sempre na parte da manhã.

A análise do padrão da marcha se deu através do protocolo de Cerny ${ }^{(16)}$ e as seguintes variáveis foram analisadas: tempo total da marcha, velocidade linear da marcha. Esse protocolo consiste em analisar a marcha no plano sagital em uma passarela de 16 metros distribuídas da seguinte forma: 1 - área inicial de 5,0 $\mathrm{m}$ de comprimento; 2 - área central 6,0 $\mathrm{m}$ de comprimento; 3 - área final de $5,0 \mathrm{~m}$ de comprimento. Não houve marcação do tempo da marcha nos 5 metros iniciais e 5 metros finais. O tempo total da marcha só foi cronometrado a partir da marcação central dos seis metros.

A mobilidade de tornozelos foi realizada através da goniometria de forma ativa para os movimentos de plantiflexão e dorsiflexão. A verificação da dorsiflexão ocorreu com o paciente sentado, com os joelhos fletidos e o pé em posição anatômica. O braço fixo do goniômetro Carci $^{\circledR}$ colocado paralelo à face lateral da fíbula, o braço móvel do goniômetro paralelo à superfície lateral do quinto metatarsal e o eixo na articulação do tornozelo junto ao maléolo lateral. A verificação da plantiflexão ocorreu com o paciente deitado em decúbito dorsal estando o pé em posição anatômica. $\mathrm{O}$ braço fixo do goniômetro ficou paralelo à face lateral da fíbula, o braço móvel paralelo à superfície lateral do quinto metatarsal e o eixo na articulação do tornozelo junto ao maléolo lateral ${ }^{(17)}$. Em ambos os movimentos, a pesquisadora solicitou a realização da amplitude máxima e o braço móvel do goniômetro era deslocado até o registro da amplitude articular, mensurado em graus.

O Grupo Controle permaneceu realizando a atividade física regular duas vezes semanais, com o profissional de Educação Física fazendo o controle da presença dessas idosas. Os exercícios englobavam exercícios de fortalecimento muscular, equilíbrio, alongamento muscular. 
Havia também um período de aquecimento e desaquecimento e a sessão durava 45 minutos. $\mathrm{O}$ GC recebeu avaliação inicial no mesmo período do grupo treinamento neuromotor e a reavaliação ocorreu após 12 semanas.

O treinamento neuromotor ocorreu ao longo de 12 semanas, com uma frequência de 2 encontros semanais, de acordo com metodologia baseada em estudos prévios ${ }^{(18-20)}$. Cada encontro durou 45 minutos com as seguintes fases: aquecimento com duração de 5 minutos contendo exercícios metabólicos de membros superiores e inferiores, treinamento neuromotor com duração de 35 minutos e desaquecimento com 5 minutos de duração com alongamentos e exercícios respiratórios de volta à calma. $\mathrm{O}$ treinamento neuromotor ${ }^{(21)}$ foi disposto em forma de circuito constituído de 10 estações (Quadro 1). As idosas repetiram 3 vezes o circuito com a permanência de 1 minuto em cada estação com 30 segundos de intervalo entre elas. O circuito continha legendas autoexplicativas com setas, pegadas e números para uma apresentação simples e didática às idosas para a execução dos exercícios. Uma pesquisadora ficava supervisionado os 5 primeiros exercícios e a outra pesquisadora supervisionava os 5 últimos exercícios do circuito.

O grau de dificuldade aumentou ao longo do treinamento a partir da sexta semana com o acréscimo de estimulações sensoriais, como, repetição de cores, números e objetos e movimentos associados de tronco e cabeça durante a realização das tarefas, sendo intercaladas em cada estação $^{(21)}$.

A análise estatística ocorreu com o auxílio do programa estatístico SPSS versão 21.0, por meio de análises descritivas, exploratórias e inferenciais conduzidas considerando-se o nível de significância de $p<0,05$. Os resultados estão apresentados com média $(\mathrm{M})$, desvio padrão (DP), frequência relativa (\%) e frequência absoluta. A normalidade dos dados foi testada através do teste de Shapiro-Wilk e a homogeneidade dos dados com o teste de Levene. A comparação entre grupos ocorreu através do teste
$\mathrm{T}$ amostras independentes, pois os dados da amostra são normais e homogêneos entre os grupos.

Após os esclarecimentos, os participantes forneceram seu consentimento para participação na pesquisa, de acordo com a Resolução $n^{\circ} 466 / 12$ do Conselho Nacional de Saúde, sendo a coleta de dados iniciada após aprovação pelo Comitê de Ética em Pesquisa da Universidade do Contestado Campus Mafra (Parecer n $\left.\mathrm{n}^{\circ} 1.022 .531 / 2015\right)$.

\section{RESULTADOS}

A presente pesquisa contou com 26 idosas distribuídas em dois grupos da seguinte forma: Grupo Controle (GC) com 15 idosas e Grupo Treinamento Neuromotor (GTN) com 11 idosas que receberam o treino neuromotor.

A média de idade do GTN foi 70,4 $\pm 3,95$ anos e do GC foi $67,9 \pm 3,51$ anos. Não houve diferença significativa entre grupos quanto à idade $(\mathrm{p}=0,43)$. A média de índice de massa corporal encontrado no GTN foi de 25,7 e no GC de 26,3 .

As características sociodemográficas das participantes da presente pesquisa estão expostas na Tabela I.

Não houve diferença significativa nos parâmetros avaliados no padrão da marcha entre os grupos (Tabela II).

De maneira geral, a amplitude de movimento de plantiflexão em ambos os tornozelos no grupo controle já era maior que o grupo treinamento neuromotor na avaliação inicial. Houve diferença significativa entre os grupos no pós-teste na dorsiflexão em ambos os tornozelos em ambos os grupos (Tabela III). Encontraram-se valores de mínimo e máximo de $10^{\circ}$ e $44^{\circ}$ para flexão plantar em ambos os tornozelos e para flexão dorsal de $6^{\circ}$ e $24^{\circ}$, como valores de mínimo e de máximo, respectivamente.

E quanto ao tempo de deambulação, na avaliação inicial dessa variável, no grupo controle foi maior $(6,68 \mathrm{~s})$ que no grupo treinamento neuromotor $(6,37 \mathrm{~s})$.

Quadro 1 - Treinamento neuromotor em forma de circuito.

\begin{tabular}{|cl|}
\hline TREINAMENTO NEUROMOTOR \\
\hline ESTAÇÕES & EXERCíCIOS \\
1 & Exercícios em apoio unipodal “avião" \\
2 & Marcha sensibilizada (com o apoio apenas dos calcanhares) de costas \\
3 & Marcha sobre superfície instável (colchão) \\
4 & Marcha com o apoio apenas do terço anterior dos pés \\
5 & Marcha com estreitamento de base em trajeto circunferencial \\
6 & Deslocamento agachado \\
7 & Passadas laterais \\
8 & Marcha de frente, pernas afastadas, com o apoio total dos pés \\
9 & Marcha de frente, pernas cruzadas, com o apoio total dos pés \\
10 & Marcha em linha reta para frente e para trás \\
\hline
\end{tabular}

Fonte: Adaptado de Costa et al. (2009) ${ }^{(21)}$. 
Tabela I - Características sociodemográficas. Mafra, SC, 2015.

\begin{tabular}{|c|c|c|c|c|c|}
\hline \multirow{2}{*}{ Variável } & \multicolumn{2}{|c|}{ GC $(n=15)$} & & \multicolumn{2}{|c|}{ GTN (n=11) } \\
\hline & $\mathrm{n}$ & $\%$ & & $\mathbf{n}$ & $\%$ \\
\hline \multicolumn{6}{|l|}{ Profissão } \\
\hline Costureira & 1 & 6,66 & Professora & 1 & 9,09 \\
\hline Atendente de açougue & 1 & 6,66 & Auxiliar & 1 & 9,09 \\
\hline Dona de casa & 1 & 66,6 & Costureira & 1 & 9,09 \\
\hline Escriturária & 1 & 6,66 & Doméstica & 2 & 18,18 \\
\hline Diarista & 1 & 6,66 & Dona de casa & 5 & 45,45 \\
\hline Servente & 1 & 6,66 & Diarista & 1 & 9,09 \\
\hline \multicolumn{6}{|l|}{ Escolaridade } \\
\hline Fundamental & 12 & 80 & & 10 & 90,90 \\
\hline Médio & 2 & 13,33 & & - & - \\
\hline Superior & 1 & 6,66 & & 1 & 9,09 \\
\hline \multicolumn{6}{|l|}{ Estado Civil } \\
\hline Casada & 5 & 33,33 & & 4 & 36,36 \\
\hline Solteira & - & - & & 1 & 9,09 \\
\hline Viúva & 10 & 66,66 & & 6 & 54,54 \\
\hline \multicolumn{6}{|l|}{ Patologias } \\
\hline Diabetes mellitus & 3 & 20 & & 4 & 25 \\
\hline Hipertensão & 10 & 66,66 & & 8 & 50 \\
\hline Cardiopatia & 2 & 13,33 & & 4 & 25 \\
\hline
\end{tabular}

GC: grupo controle; GTN: grupo de treinamento neuromotor.

Tabela II - Padrão da marcha. Mafra, SC, 2015.

\begin{tabular}{lccc}
\hline \multirow{2}{*}{ Padrão da marcha } & \multicolumn{2}{c}{ Grupos } & \multirow{2}{*}{ valor de $\mathbf{p}^{*}$} \\
\cline { 2 - 3 } Velocidade (m/s) & GC (n=15) & GTN (n=11) & 0,55 \\
$\quad$ Pré & $0,93 \pm 0,21$ & $0,98 \pm 0,22$ & 0,53 \\
Pós & $0,97 \pm 0,22$ & $1.03 \pm 0.24$ & 0,58 \\
Tempo de deambulação (segundos) & & & 0,60 \\
Pré & $6,68 \pm 1,30$ & $6,37 \pm 1,55$ & \\
Pós & $6,39 \pm 1,24$ & $6,10 \pm 1,54$ & \\
\hline
\end{tabular}

Resultados estão descritos como média \pm desvio padrão. GC: grupo controle e GTN: grupo treinamento neuromotor. *Teste t independente.

Tabela III - Mobilidade articular de tornozelos. Mafra, SC, 2015.

\begin{tabular}{lccc}
\hline \multirow{2}{*}{ Goniometria (graus) } & \multicolumn{2}{c}{ Grupos } & \multirow{2}{*}{ valor de $\mathbf{p}^{*}$} \\
\cline { 2 - 3 } Plantiflexão direito & GC (n=15) & GTN (n=11) & $0,04^{*}$ \\
$\quad$ Pré & $33,20 \pm 6,17$ & $26,45 \pm 10,21$ & 0,28 \\
$\quad$ Pós & $34,20 \pm 6,37$ & $29,91 \pm 13,30$ & $0,03^{*}$ \\
Plantiflexão esquerdo & & & 0,17 \\
$\quad$ Pré & $34,60 \pm 6,13$ & $27,45 \pm 9,66$ & 0,17 \\
$\quad$ Pós & $35,13 \pm 6,19$ & $30,18 \pm 11,68$ & $0,02^{*}$ \\
Dorsiflexão direito & $19,07 \pm 3,53$ & $16,91 \pm 4,41$ & $0,01^{*}$ \\
$\quad$ Pré & $19,67 \pm 2,92$ & $16,73 \pm 3,25$ & $0,00^{*}$ \\
$\quad$ Pós & & & $13,18 \pm 4,40$ \\
Dorsiflexão esquerdo & $18,67 \pm 2,58$ & $13,55 \pm 4,27$ & \\
$\quad$ Pré & $18,93 \pm 1,98$ & & \\
Pós &
\end{tabular}

Resultados estão descritos como média \pm desvio padrão. GC: grupo controle e

GTN: grupo treinamento neuromotor. 


\section{DISCUSSÃO}

Os resultados encontrados na presente pesquisa mostram que a atividade física regular e o treinamento neuromotor favorecem a manutenção do bom desempenho da marcha e mobilidade de tornozelos em idosos.

A prática da atividade física regular incide beneficamente nas alterações decorrentes do processo de envelhecimento, auxiliando a manutenção das funções, isso pode contribuir para manter e/ou melhorar a força, a flexibilidade, a coordenação e o equilíbrio, elementos da aptidão física essenciais para manter a capacidade funcional no idoso ${ }^{(22)}$.

Há um declínio não linear no equilíbrio, força muscular e na marcha com o envelhecimento, o que justifica a necessidade de se preservar os fatores que contribuem para uma maior independência funcional. A manutenção da força muscular dos membros inferiores, uma marcha segura e eficiente e um bom equilíbrio podem afetar positivamente a saúde e a independência funcional dos idosos, pois quando estes apresentam uma disfunção do equilíbrio, uma pobre marcha e reduzida força muscular aumentam-se os riscos de quedas e fraturas ${ }^{(23)}$.

No entanto, observando-se o princípio da especificidade do treinamento, para o desenvolvimento da velocidade da marcha, os exercícios específicos de funcionalidade foram superiores ao treinamento geral de força, embora os dois treinamentos tenham sido eficientes. Posto isso, nenhum exercício físico parece ser melhor do que a prática da própria marcha para o desenvolvimento do desempenho funcional da marcha em idosos ${ }^{(24)}$. Embora, na presente pesquisa, o treino neuromotor fosse composto de diversos exercícios, nenhum deles era especificadamente focado para melhora direta da velocidade da marcha.

Há uma relação positiva entre exercício físico e desempenho da marcha em idosos saudáveis. Dessa maneira, é possível afirmar que a diminuição que ocorre com o passar dos anos de certas capacidades, essenciais para um bom desempenho da marcha como força, equilíbrio, coordenação, flexibilidade e potência, pode ser compensada em diferentes graus com o exercício físico ${ }^{(25,26)}$.

No presente estudo, na avaliação inicial da variável tempo de deambulação, o grupo controle foi maior $(6,68 \mathrm{~s})$ que no grupo treinamento neuromotor $(6,37 \mathrm{~s})$. Contrastando com uma pesquisa que comparou um grupo praticante de musculação $(4,87 \mathrm{~s})$ e outro grupo sedentário $(5,65 \mathrm{~s})$, onde mostrou que indivíduos sedentários tiveram um tempo maior de deambulação(27).

Um estudo realizado com idosas saudáveis que receberam um programa de exercícios contendo exercícios aeróbios, equilíbrio, coordenação, alongamentos e treino de marcha com o objetivo de melhorar o desempenho físico associado à cinemática da marcha, revelou que houve uma diminuição na velocidade da marcha após 12 semanas (de $1,37 \mathrm{~m} / \mathrm{s} \pm 0,15$ para $1,28 \mathrm{~m} / \mathrm{s} \pm 0,28)^{(28)}$. No presente estudo, não houve um treino específico de marcha na aplicação do treinamento neuromotor, podendo ser um fator que contribuiu para que os resultados não fossem significativos para o padrão de marcha das idosas avaliadas.

Há uma relação linear entre a idade e a flexibilidade articular em idosos. Uma pesquisa verificou a relação entre idade dos idosos e flexibilidade de tornozelos e os resultados demonstraram diferença estatística quando comparado a faixa etária de 65 a 69 anos, 70 a 74 anos, 75 a 79 anos quando comparados entre si e com os grupos com mais de $80 \operatorname{anos}^{(29)}$. Os achados deste estudo corroboram com o perfil da amostra estudada na presente pesquisa, pois a média de idade nos dois grupos foram $67.93 \pm 3.51$ anos no grupo GC e $70.45 \pm 3.95$ anos no grupo GTN.

A partir de um estudo de revisão com o objetivo de analisar velocidade da marcha e sobrevida utilizando estudos que reuniram 9 estudos de coorte com um total de 34.485 idosos ( $73,5 \pm 5,9$ anos) da comunidade e a velocidade média da marcha encontrada foi de $0,92 \mathrm{~m} / \mathrm{s}$ e que há relação entre a velocidade da marcha e sobrevida e está diretamente relacionada com o avançar da idade ${ }^{(30)}$. A presente pesquisa demonstrou que os resultados encontrados estão dentro dos valores usuais para esta faixa etária.

Um estudo $^{(31)}$ de coorte com 3.042 idosos da comunidade definiu, como ponto de corte da velocidade da marcha usual em idosos, como valor preditivo para eventos relatados de saúde, de $1 \mathrm{~m} / \mathrm{s}$ para 6 metros de distância percorrida na avaliação da velocidade da marcha, independente de sexo e diferenças de raça. Os valores da velocidade da marcha encontrados em ambos os grupos da presente pesquisa estão muito próximos de $1 \mathrm{~m} / \mathrm{s}$, portanto é importante que idosos sejam acompanhadas com avaliações sistemáticas do padrão e da cinemática da marcha.

A mobilidade normal de tornozelo está intimamente ligada às atividades de vida diária que requerem o controle postural dinâmico do indivíduo sofrendo constantes desgastes que se tornarão maiores e mais perceptíveis com o avançar da idade, comprometendo assim a mobilidade dessa articulação(32). Em um estudo com 30 idosas sedentárias, com idade média de 77,48 $\pm 6,94$ anos, onde foi mensurada a amplitude de flexão plantar e de flexão dorsal bilateral, mostrou que as angulações mínima e máxima para flexão plantar no tornozelo direito foram de $10^{\circ}$ e $45^{\circ}$, respectivamente, e no tornozelo esquerdo esses valores foram de $18^{\circ}$ e $45^{\circ}$, respectivamente. Já a variável 
flexão dorsal direita e esquerda obteve resultado mínimo e máximo, respectivamente, $6^{\circ}$ e $20^{\circ(33)}$. No presente estudo, encontraram-se valores de mínimo e máximo de $10^{\circ}$ e 44 ${ }^{\circ}$ para flexão plantar em ambos os tornozelos e para flexão dorsal de $6^{\circ}$ e $24^{\circ}$ como valores de mínimo e máximo respectivamente.

A associação entre a idade e amplitude de movimento das articulações tem sido investigada para determinar as limitações impostas pelo avançar da idade. Um estudo observacional revelou que a amplitude articular de plantiflexão do tornozelo encontrada para a faixa etária de 65 a 69 anos foi de $21,67 \pm 5,59$ graus e na faixa etária de 70 a 74 anos foi de $23,75 \pm 7,44$ graus ${ }^{(29)}$. No presente estudo, encontraram-se valores superiores no movimento de plantiflexão de tornozelos $(\mathrm{GC}=33,20 \pm 6,17$ e $\mathrm{GTN}=$ $26,45 \pm 10,21$ graus).

Dentro do contexto que envolve o envelhecimento saudável, a manutenção de altos níveis de habilidades funcionais deve ser prioridade no processo de promoção à saúde do idoso. A política pública de saúde do idoso parte do pressuposto de que, para se envelhecer de forma saudável e bem-sucedida, é preciso favorecer oportunidades para que os indivíduos possam optar por estilos de vida saudáveis e, ainda, fazer controle do próprio status de saúde ${ }^{(34)}$. Cabe aos profissionais da saúde participarem do processo, independente do ambiente profissional em que estiverem inseridos. Sendo assim, poder-se-á favorecer o envelhecimento como um processo positivo em relação às modificações musculoesqueléticas que ocorrem na senescência.

O presente estudo apresenta como limitação o tamanho reduzido da amostra, não permitindo a generalização dos resultados. Ainda, propõe-se que novos estudos sejam realizados com a avaliação de outros aspectos do padrão de marcha em idosos, bem como, sejam utilizados outros instrumentos quantitativos dos parâmetros cinemáticos da marcha.

\section{CONCLUSÃO}

$\mathrm{O}$ estudo demonstrou que o treinamento neuromotor beneficiou a manutenção do padrão de marcha (velocidade e tempo de deambulação) e a mobilidade articular de tornozelos em idosas avaliadas.

\section{REFERÊNCIAS}

1. Ferreira FPC, Bansi LO, Paschoal SMP. Serviços de atenção ao idoso e estratégias de cuidado domiciliares e institucionais. Rev Bras Geriatr Gerontol. 2014;17(4):911-26.

2. Sousa SPO, Branca SBPB. Panorama epidemiológico do processo de envelhecimento no mundo no Brasil e Piauí: evidências na literatura. Enferm Foco (Brasília). 2011;2(3):188-90.

3. Instituto Brasileiro de Geografia e Estatística - IBGE. Sinopse do Senso Demográfico de 2010. Rio de Janeiro: IBGE; 2011.

4. American College of Sports Medicine, ChodzkoZajko WJ, Proctor DN, Fiatarone Singh MA, Minson CT, Nigg CR et al. American College of Sports Medicine position stand: exercise and physical activity for older adults. Med Sci Sports Exerc. 2009;41(7):1510-30.

5. Ferreira OGL. Envelhecimento Ativo e Sua Relação com a Independência Funcional. Texto \& Contexto Enferm. 2012;21(3):513-8.

6. Mann L, Kleinpaul J, Teixeira CS, Rossi AG, Lopes LFD, Mota CB. Investigação do equilíbrio corporal em idosos. Rev Bras Geriatr Gerontol. 2008;11(2):155-65.

7. Ministério da Saúde (BR). Política Nacional de Saúde da Pessoa Idosa - PNS. Brasília: Ministério da Saúde; 2010.

8. Lenardt MH, Carneiro NHK, Betiolli SE, Ribeiro DKMN, Wachholz PA. Prevalence of pre-frailty for the component of gait speed in older adults. Rev Latinoam Enferm. 2013;21(3):734-41.

9. Lima AP, Cardoso FB. Avaliação da eficácia de um programa ludomotor de exercícios físicos na melhora da capacidade funcional de idosos. Estud. Interdiscip Envelhec. 2011;18(2):429-40.

10. Pedrinelli A, Garcez LE, Nobre RSA. O efeito da atividade física no aparelho locomotor do idoso. Rev Bras Ortop. 2009;44(2):96-101.

11. Brown M, Sinacore DR, Ehsani AA, Binder EF, Holloszy JO, Kohrt WM. Low-intensity exercise as a modifier of physical frailty in older adults. Arch Phys Med Rehabil. 2000;81(7):960-5. 
12. Kovács E, Prókai L, Mészáros L, Gondos T. Adapted physical activity is beneficial on balance, functional mobility, quality of life and fall risk in communitydwelling older women: a randomized singleblinded controlled trial. Eur J Phys Rehabil Med. 2013;49(3):301-10.

13. Cruz A, Oliveira EM, Melo SIL. Análise biomecânica do equilíbrio do idoso. Acta Ortop Bras. 2010;18(2): 96-9.

14. Guimarães JMN, Farinatti PTV. Descriptive analysis of variables theoretically associated to the risk of falls in elder women. Rev Bras Med Esporte. 2005;11(5):299305.

15. World Health Organization-WHO. World report on Ageing and Health 2015.Geneva: WHO; 2015.

16. Henriques GRP, Ribeiro ASB, Corrêa AL, Sanglard, RCF, Pereira, JS. A interferência da redução progressiva nas amplitudes da articulação coxofemural na velocidade da marcha. Fit \& Perfor Jour. 2003;2(3):183-9.

17. Marques AP. Manual de goniometria. São Paulo: Manole; 2003.

18. Farinatti PTV, Lopes LNC. Amplitude e cadência do passo e componentes da aptidão muscular em idosos: um estudo correlacional multivariado. Rev Bras Med Esporte. 2004;10(5);389-94.

19. Ribeiro ASB, Pereira JS. Melhora do equilíbrio e redução da possibilidade de queda em idosas após os exercícios de Cawthorne e Cooksey. Rev Bras Otorrinolaringol. 2005;71(1):38-46.

20. Zambaldi PA, Costa TABN, Diniz GCLM, Scalzo PL. Efeito de um treinamento de equilíbrio em um grupo de mulheres idosas da comunidade: estudo piloto de uma abordagem específica, não sistematizada e breve. Acta Fisiatr. 2007;14(1):17-24.

21. Costa JNA, Gonçalves CD, Rodrigues GBA, Paula AP, Pereira MM. Exercícios multissensoriais no equilíbrio e na prevenção de quedas em idosos. EFDeportes.com [Internet]. 2009 [acesso em 2015 Fev 11];14(135):119. Disponível em: http://www.efdeportes.com/efd135/ exercicios-multisensoriais-em-idosos.htm

22. Gomes M Neto, Castro MF. Comparative study of functional independence and quality of life among active and sedentary elderly. Rev Bras Med Esporte. 2012;18(4):234-37.

23. El Haber N, Erbas B, Hill K, Wark J. Relationship between age and measures of balance, strength and gait: linear and non-linear analyses. Clin Sci. 2008;114(12):719-27.

24. Krebs DE, Scarborough DM, McGibbon CA. Functional vs. strength training in disabled elderly outpatients. Am J Phys Med Rehabil. 2007;86(2): 93-103.

25. Brandalize D, Almeida PHF, Machado J, Endrigo R, Chodur A, Israel VL. Effects of differents schedule of exercise on the gait in healthy elderly: a review. Fisioter Mov. 2011;24(3):549-56.

26. Fernandes AMBL, Ferreira JJA, Gomes LROS, Brito GEG, Clementino ACCR, Souza NM. Effects of physical training on gait performance and functional mobility in elderly. Fisioter Mov. 2012;25(4):821-30.

27. Silva GG, Silva AC, Soares AS, Avellar MC, Miranda VCR. Análise biomecânica da marcha e capacidade funcional de idosos praticantes e não praticantes de musculação. Pesquisa Edu Física. 2012;11(3):17-24.

28. Cao ZB, Maeda A, Shima NK, Nishizono H. The effect of a 12-week combined exercise intervention program on physical performance and gait kinematics in community-dwelling Elderly Women. J Physiol Anthropol. 2007;26(3):325-32.

29. Schenatto P, Milano D, Berlezi EM, Bonamigo ECB. Relação entre aptidão muscular e amplitude articular, por faixa etária, na marcha do idoso. Rev Bras Geriatr Gerontol. 2009;12(3):377-89.

30. Studenski S, Perera S, Patel K, Rosano C, Faulkner K, Inzitari $\mathrm{M}$, et al. Gait speed and survival in older adults. JAMA. 2011;305(1):50-8.

31. Cesari M, Kritchevsky SB, Penninx BW, Nicklas BJ, Simonsick EM, Newman AB, et al. Prognostic value of usual gait speed in well-functioning older people. J Am Geriatr Soc. 2005;53(10):1675-80.

32. Lopes KT, Costa DF, Santos LF, Castro DP, Bastone AC. Prevalência do medo de cair em uma população de idosos da comunidade e sua correlação com mobilidade, equilíbrio dinâmico, risco e histórico de quedas. Rev Bras Fisioter. 2009;13(3):223-9. 
33. Barros JFP, Rodrigues ER, Filho VD, Fidelis CA. Correlação entre amplitude de movimento da articulação talocrural e equilíbrio estático e dinâmico de idosas de um grupo da terceira idade. Ter Man. 2011;9(44):429-33.

34. Silva HS, Lima AMM, Galhardoni R. Envelhecimento bem-sucedido e vulnerabilidade em saúde: aproximações e perspectivas. Interface Comun Saúde Educ. 2010;14(35):867-77.
Endereço do primeiro autor:

Bruna Carolina de Lima

Universidade do Contestado Mafra - SC

Avenida Presidente Nereu Ramos, 1071

Bairro: Jardim Moinho

CEP: 89300-000 - Mafra - SC - Brasil

E-mail: brunacarolinel@hotmail.com

Endereço para correspondência:

Malu Cristina de Araújo Montoro Lima

Universidade do Contestado Mafra - SC

Avenida Presidente Nereu Ramos, 1071

Bairro: Jardim Moinho

CEP: 89300-000 - Mafra - SC - Brasil

E-mail: malumontoro@gmail.com 